\title{
Editorial
}

\section{Pandemic Pause: A Mixed Blessing}

\section{Dinesh Kadam ${ }^{1}$}

\author{
${ }^{1}$ Department of Plastic and Reconstructive Surgery, A. J. Institute of \\ Medical Sciences and A J Hospital and Research Centre, Mangalore, \\ Karnataka, India
}

Indian J Plast Surg:2020;53:169-170

With no visible signs of relenting, the clutches of the pandemic are proving to be debilitating and manifestly fatal to the medical professionals. While serving, several hundreds of doctors have succumbed, and a sense of uncertain morbidity prevails among the survivors. One among the worst affected nations, India has several plastic surgeons who are an integral part of managing SARS-CoV-2 patients, and few of them are at the helm of facilities such as state-run public hospitals. Postgraduates across the specialty form the frontline workforce directly involved in treating SARS-CoV-2 patients. Besides the risks of contracting the infection, for the most the burden of economic loss is sudden and devastating. Nevertheless, the pandemic seems to have paused the world for good. While this is seemingly protracted and deeply crippling, there are a few silver linings to take away in the form of innovative strategies which are adaptive to change. It is worthwhile to reflect deeply upon how the pandemic has bestowed mixed blessings upon us as a community of plastic surgeons amidst a grim global crisis.

The initial phase of lockdown, when the pandemic in the country was limited to just a few hundreds of cases, was a welcome break for everyone-quality time for the family was assured and it also turned out to be an opportunity for personal reorganization. Trained to plan methodically, for a change, "reverse planning" was not an option! Many seized this gifted break, albeit obligatory, in a constructive manner to upregulate their skills and knowledge, and to pursue long-pending interests or passions.

Even before the countrywide lockdown, Dr. R.K. Khazanchi, the president, held a day-long APSI Executive Council meeting via Zoom ( - Fig. 1). Taking this already-in-place technology and logistics forward, APSI took up the early initiative of organizing online webinars. A surge of webinars and continuing medical education (CME) has unearthed new speakers and resource persons who could conceptualize and conduct such events. Deliberating at leisure, but in a planned manner, and participating from one's personal space was refreshing, enriching, and productive. It involved engaging discussions with the enthusiastic participation of online participants.

Address for correspondence

Dr. Dinesh Kadam, MS, DNB, MCh., Department of Plastic and

Reconstructive Surgery,

A. J. Hospital and Research Centre, Mangalore-575004, Karnataka, India (e-mail: drkadam@yahoo.co.in).

Such keynotes otherwise would have had to get packed in time-constrained "plenary sessions" of a conference.

Several specialty societies such as IAAPS, ISRM, ISCLPCA, Rhinoplasty Society, the state chapters of Karnataka, West Bengal, Rajasthan, Tamil Nadu, and institutions such as Tata Memorial Hospital, Mumbai, AFMC, Pune took part in this academic feast. Furthermore, instructional videos by YouTubers, and online learning and interactive platforms such as Plasticos Network, Young Plastic Surgeons (YPS) web group, International Microsurgery Club gained immense popularity over the course of these sessions. Notably, for once, members of the various subspecialties of plastic surgery found a common meeting ground and audience, irrespective of their affiliations.

A plethora of online teaching sessions, explicitly designed for postgraduates in particular, heralds a new era of plastic surgery education. Teachers-cum-examiners of repute and experts within the field led the case discussions to benefit students on the verge of appearing for examinations. The efforts of Plastiquest, Aplast, and Calplast are indeed laudable for their innovative ideas, encompassing all postgraduates, at no cost, breaking away from the earlier 2-day distant paid courses. The entire content was further made available on YouTube and The MediTube. The digital format was a natural transformation for tech-savvy postgraduates. The benefits are enriching to the students and teachers alike.

The lockdown led many to explore even Instagram and Twitter, which are now pervasive in the this era of social and professional life. The benefits of these popular and powerful social media platforms, which are multifunctional in nature, providing learning, leading to interaction, and serving as a tool of advocacy, cannot be overemphasized.

Extended social distancing and injunction on public meetings has now pushed the scientific societies to explore and resort to virtual meetings and conferences. Ever innovative, The Ganga Medical Center, Coimbatore, held a successful online operative workshop, a fine example to emulate. Despite the lack of personal interaction and the warm hospitality of regular meetings, the pros outweigh the cons in terms of the cost, travel, time, and logistics: a new reality and new norms of future academics.

(0)2020 Association of Plastic Surgeons of India
License terms

10.1055/s-0040-1716612 ISSN 0970-0358. 


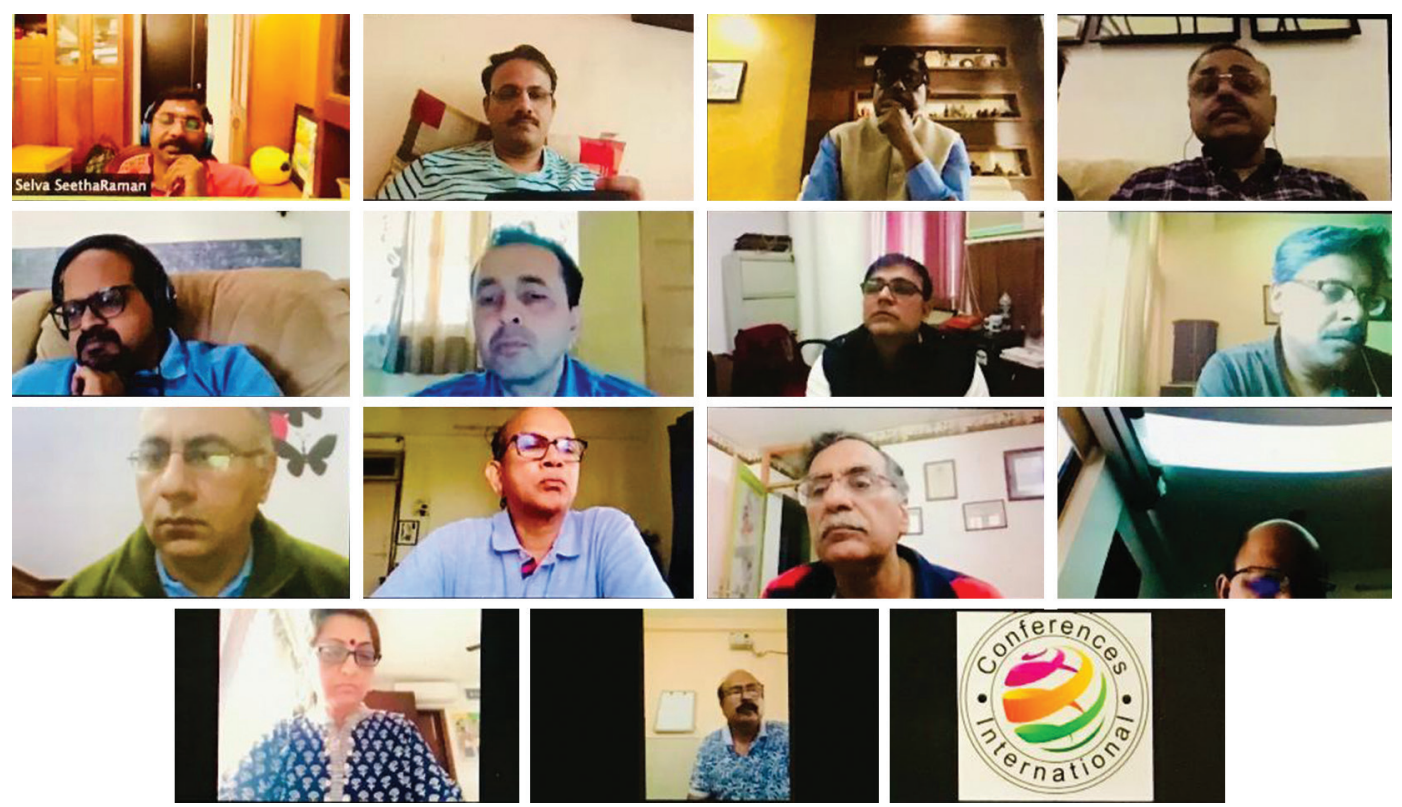

Fig. 1 APSI-EC virtual meeting held on March 15, 2020. (Image courtesy: Dr. Nilesh Satbhai.)

Mulling over proceedings carefully, and left with no viable alternatives, the APSI has declared 2020 as a "zero year" for all purposes, including all accredited events, conferences, elections, fellowship programs, and so on. This again is a mixed blessing, where we stand to lose organized and fruitful socioprofessional activities; however, financially, it is a great "saver year," unless, of course, APSICON2020 brought along more savings if it were to be held. Virtual executive meetings have incurred no expense to the APSI as have the expenditures pertaining to regular activities. Our journal remains the most suitable option to obtain much on account of these savings.

It is a mixed blessing for the IJPS, too, with submissions galore, but at the cost of substantial revenue loss due to the current financial slowdown. A staggering $200 \%$ increase in articles during this "zero year" is a much-desired impetus for progress. This propulsion, I believe, will lead to a regular flow of a high volume of articles in the coming days. Editors, reviewers, and publisher have their work cut out. I am glad to announce that, from 2021, we will publish four issues a year. This increase in long-awaited frequency will be fulfilled despite the precarious financial situation. Sustaining financial stability for the journal in the current context is deeply concerning and exceptionally challenging.
Much effort has gone in for timely publication of each issue during this countrywide paralysis. The shutdown of production houses and the disruption of the supply chains have hit the printing and delivery system hard. We sincerely apologize for this inevitable delay.

Finally, the scale of the pandemic has tested the resilience and survival instincts of the specialty. A wide range of problems, including emergencies, trauma, malignancy, burns, reconstructive procedures, and so on, were attended to in most places, although the volume was comparatively lesser. Many aesthetic surgeons have resumed their work with safety protocols in place. Remaining steadfast is testament to the scope and diversity of the specialty, even in times of adversities of this magnitude. It perhaps makes a strong case of attracting an increasing number of young aspirants to pursue this career.

Amidst a global crisis, the pandemic has brought a forward vision along with a growing acceptance toward many progressive changes. It could be a watershed moment, infusing new thinking. Undeniably, with several positives and steps that permeate, it could possibly define the future course of APSI and the medical community at large.

\section{Conflicts of Interest}

None declared. 